\title{
Collaborative engineering as a basic concept for teaching structuration
}

\author{
A. Bernard* \\ IRCCyN, Ecole Centrale de Nantes, \\ Nantes, France \\ E-mail: alain.bernard@ec-nantes.fr \\ ${ }^{*}$ Corresponding author
}

\section{N. Perry}

LGM $^{2} \mathrm{~B}$, Bordeaux 1 University, Bordeaux, France

E-mail: nicolas.perry@iut-bordeaux1.fr

\section{P. Dépincé}

IRCCyN, Ecole Centrale de Nantes,

Nantes, France

E-mail: philippe.depince@ec-nantes.fr

\begin{abstract}
Engineering education is evolving in a global context. Projects activities allow students structuring and applying the knowledge learned while facing problems. But, when taking into account MultiDisciplinary Optimisation (MDO), it is necessary to model and to exploit the all context and technical constraints. Two elements lead such approach. First of all, roadmaps give structured processes for the resolution of project objectives. Then, MDO allows solving local problems. The collaborative engineering needs to achieve the final choices based on the indications of strategic inputs, roadmaps and MDO results. This paper relates concrete experiences and proposes some progress for future engineering education.
\end{abstract}

Keywords: collaborative engineering; decision making; MDO; multidisciplinary optimisation, teaching.

Reference to this paper should be made as follows: Bernard, A., Perry, N. and Dépincé, P. (2009) 'Collaborative engineering as a basic concept for teaching structuration', Int. J. Collaborative Engineering, Vol. 1, Nos. 1/2, pp.168-184.

Biographical notes: Professor Alain Bernard, graduated in 1982, obtained his $\mathrm{PhD}$ in 1989. As an Assistant-Professor, he worked from 1990 to 1996, in Ecole Centrale de Paris, on product, technology and process modelling. From September 1996 to October 2001, he has been Professor in CRAN, as the Head of the Mechanical and Production Engineering team. His main research topics are related to RE, KBS, CAPP, product and process modelling, integration of economical and human aspects. His actual position is Professor and Deputy Director for Research at Ecole Centrale de Nantes, and at IRCCyN (Head of the "Virtual Engineering for industrial engineering" project).

In $2000 \mathrm{~N}$. Perry received his $\mathrm{PhD}$ in Mechanical Engineering at Nantes University, focusing on the material - process interaction. From 2001 to 2007 , 
in the Research Institute on Communications and Cybernetics of Nantes (IRCCyN), research topics focused on performance improvement using Virtual Engineering approaches. Knowledge Management is the used to define Knowledge Based Environments as Engineers Decision Tools in the area of Costs and Value Management. At Bordeaux1 University, in the Mechanical Engineering and Materials Laboratory $\left(\mathrm{LGM}^{2} \mathrm{~B}\right)$, research topics moved to composites domain, completing mechanic and economic to ecologic interests. The integration from the design to the process allows facing simulation and experimentation to evaluate design and industrial impacts.

Philippe Dépincé is Professor at the Ecole Centrale de Nantes (France). He obtained the Engineering Degree in 1990 and received a PhD in Mechanical Engineering from the Ecole Centrale de Nantes in 1993. He is a researcher at the Cybernetic and Communication Research Institute of Nantes (IRCCyN) since 1994 and his research interests are linked to Design and Manufacturing processes and includes, among other fields, computer aided process planning and design optimisation (AI tools such as Multi-Agents Systems and Decision aided, multidisciplinary and multicriteria optimisation).

\section{New goals for engineering education}

Engineering education is evolving in a global context, mainly due to two different reasons. The first one comes from the internationalisation of the student groups attending engineering studies in a 'double diploma' context or for specialisation. The second is related to the development of mecatronic integrated systems, and moreover, of multi-technologies complex systems, like automobiles, planes, ships, etc. In such systems, electronics control the behaviour of the complex system that is also constituted of parts, components and subsystems that are most often seen as 'functional units' and defined by their ability to deliver given outputs corresponding to given inputs.

So, in such a context, it is necessary to extend the classical lectures to socio-technical and economical aspects to be able to globally consider the systems while being able, at the design level, to define a common base of understanding for the different experts involved in the design process. But a common base is not enough if it is limited to an information database where each of the actors can put and get the information he would need based on his skills. It is also necessary to define and manage the design process in the same way, which means that the different actors have to improve their own efficiency by collaborating. But most often, such collaboration introduced the necessity to manage at the same time different constraints from the different technical domains addressed within the design process. So, this increases the complexity of the design process when letting the actors have access to different information more rapidly and efficiently. This constitutes a compromise that has to be considered as a very interesting opportunity for the design process actors.

The goal at the end is to let the different experts adopt a way of collaborative engineering, not only by assuming both their own constraints but also by contributing to the global efficiency of the group and a better result of the design. This result is a complex system that has better chances to fit the life-cycle requirements and that would not necessitate numerous modification loops at industrialisation stage for improving its performances. 
The consequence for the education curriculum derives from these considerations. Based on the previous aspects, training is driven by two aspects, of course the knowledge of fundamental scientific fields (like mathematics, physics, etc.) and the experience of system approach in a pedagogy based on projects.

The objectives of this paper are first to give general bases of these considerations, particularly activity theory as a base of the approach of each-other interaction during project discussions, and also multi-disciplinary optimisation as the base of problem-solving and decision-making, and second to describe and argue an example of engineering education program, which is based on this approach. It will be shown how laboratory research activities and experiments and also platform-based projects can be profitable for the efficiency of the education of engineering students.

The first part will highlight some bases from collaborative engineering, more precisely Engineering as Collaborative Negotiation (ECN) initial concept proposed by Professor S. Lu. The second part allows giving some very few elements on activity theory, which can be considered as a base for positioning the context both of teaching and of practising collaborative engineering during projects, in the general social environment. The third part will introduce the project-based pedagogy as a way of experimenting this way of working. An example of a collaborative engineering platform, EDEN, is presented. In the fourth part, the necessity of multi-domain optimisation, which is complementary to the functions of the previous platform, is demonstrated as a way for improving the collaboration in the sense of giving elements for a computer-aided decision-making. Some recent research results are introduced. Finally, a discussion and conclusion section summarises the content of the paper and proposes some way of progress for the future of engineering education.

\title{
2 Collaborative engineering paradigm
}

During the past years, Professor S. Lu defined and detailed the basic concepts of collaborative engineering. He proposed different seminars where different experts had the opportunity to give their point-of-view of this paradigm.

One of the conclusions of initial discussions is that this paradigm should have a significant influence on education contents and methods. The following sentence is from the introductive paper elaborated before the ECN seminar of Shanghai in 2005:

\begin{abstract}
"Second, the ECN study treats collaborative engineering as a human activity within a socio-technical environment. Here, an activity is formally defined according to the Cultural Historical Activity Theory (CHAT) as a conscious human endeavour driven by goals and motives. This classic psychology theory suggests that interactive human activities include three types: coordination (e.g., plan each others' activities in case of sequential dependency), cooperation (e.g., taking each others decisions into consideration in case of reciprocal reliance), and construction (e.g., develop new understanding and solutions in case of shared knowledge). We believe that engineering is more about the construction type of human interaction, and the group construction (or co-construction) activity in engineering is best achieved by collaborative negotiation."
\end{abstract}

To go further in the way of clarifying and demonstrating the basic concepts, it is also important to have in mind the context of the studies we consider: engineering. 
If one refers to the National Collaborative for Engineering Graduate Education Reform (USA, 2003), it can be considered that

\begin{abstract}
"Engineering is a creative profession, concerned, with the combining of human, material and economic resources to meet the needs of society ... for the advancement and betterment of human welfare." (National Collaborative Task Force, September 19, 2003)
\end{abstract}

One of the results proposed in this context was some recommended course topics, which were the following ones:

Curriculum requirements: Math requirements, Engineering management requirements. The core curriculum requirements can be summarised by the six following items: Systems engineering, Software engineering, Manufacturing processes, Engineering disciplines (electrical, mechanical, chemical, aeronautics), Decision-making and problem-solving, Business fundamentals (finance, marketing, accounting, supply chain). An elective module could be proposed, defined for each of the engineering disciplines by the industry and university partnerships. These considerations can be summarised in Figure 1.

Figure 1 The well-rounded engineer (see online version for colours)

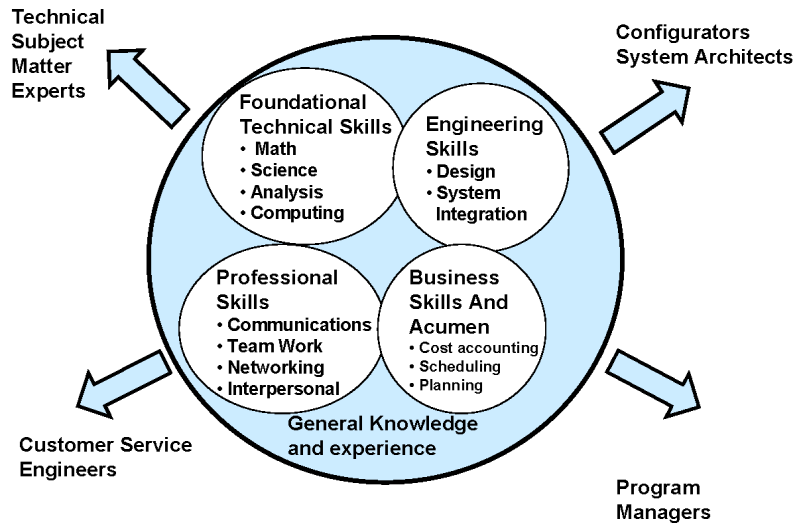

Source: Engineering education objective, the well-rounded engineer, by Dr. John McMasters, AIAA paper 2004

\title{
3 Activity theory as the base for fundamental concepts of collaborative engineering
}

As previously mentioned, the activity concept has been formally defined by different communities but one can refer to the CHAT as "a conscious human endeavour driven by goals and motives". Humans interact with other humans and also with complex systems, but always in a socio-technical context.

Different authors have proposed some definitions about activity theory. Kari Kuutti considers that

\footnotetext{
"activity theory is a philosophical and cross-disciplinary framework for studying different forms of human practices as development processes, with both individual and social levels interlinked at the same time."
} 
Victor Kaptelenin addresses the following comment:

"The general philosophy of activity theory can be characterised as an attempt to integrate three perspectives: the objective, the ecological and the socio-cultural. Like cognitive psychology, activity theory ... is a "natural science like" like theory."

Another point of view is proposed by Bonnie Nardi who considers that

"Activity theory is a powerful and clarifying descriptive tool rather than a strongly predictive theory. The object of activity theory is to understand the unity of consciousness and activity."

On a global point of view, the psychology theory suggests that interactive activities are of three types: coordination because of dependencies, cooperation to take into consideration other decisions in case of reciprocal reliance, and construction by developing new understanding and solutions based on shared knowledge. Engineering activities are related to these three levels but more directly visible as a construction achievement, and most often, a co-construction achievement. What is assumed here is that the co-construction has major benefit in achieving collaborative negotiation as a way to obtain the best result in solving problems. The major components that characterise the activity theory model are given in Figure 2. In the following, these features are not going to be detailed and one can refer to the literature to go deeper in this theory.

Figure 2 A representation of basic concepts of the activity theory model (see online version for colours)

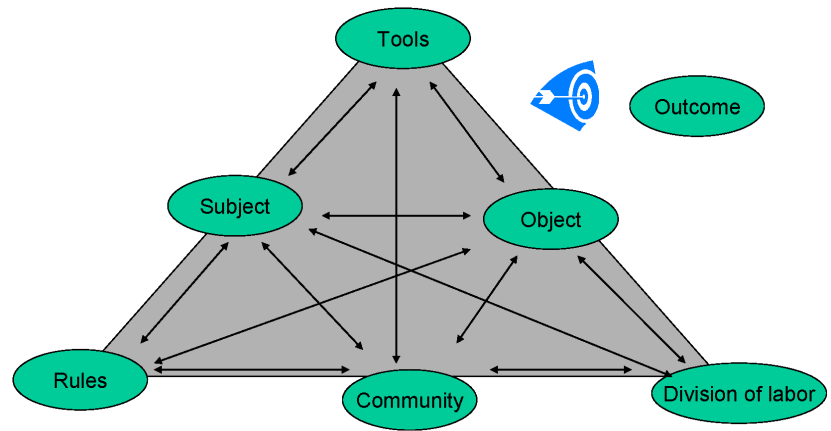

Based on basic elements and considering that the Activity theory can be valuable for the structuration of the curricula of engineering education, this is fundamental to consider such ideas when elaborating a teaching strategy. It is necessary for the students to practice such approach and to utilise such concepts even if they do not have the financial pressure. They most often have the time pressure and all of the students do not have the same competencies and also do not have the same goals. Buccarelli game is one of the most well-known games that enables students to collaborate to achieve a project in a minimum of time and also that rich as much as possible the requirements of each of the participants. Collaborating negotiation is then indispensable to be efficient due to the limited time.

But when the engineering students face a real design problem in a real project context, they have to manage a large volume of information, to interact with many more other actors and to proceed for many decisions based on different knowledge. 
In this case, and mainly due to the extended enterprise reality, it is of benefit for the students to have the benefit of using platforms enabling collaborative engineering. This will not really help for collaborative negotiation but this kind of platform will structure the information related to the projects, the actors, the activities and associated documents and standards. In our case, we have considered that during their studies, the student should be able to structure their project methods and to apply a collaborative strategy as this will be the case during their future jobs. We are convinced of the benefits of a pedagogy based on projects, and moreover, when it is possible, a pedagogy utilising collaborative engineering platforms and also multi-disciplinary methods and tools that help them in problem-solving and decision-making. These different aspects are presented in the following sections.

\section{$4 \quad$ Pedagogy based on projects}

\subsection{Project-based approach: structuration, capitalisation and traceability}

Education based on projects let students have the opportunity to apply the different theories and methodologies on practical collaborative activities and to have very valuable feedbacks from their own experiences about these projects.

When considering the industrial needs, it is confirmed that industry, and more widely, companies need some engineers with complementary know and know-how. Such a demand is based on a bi-polar approach, based on fundamentals in scientific and technological courses, and also on transversal and practical applications, focused on industrial demands and goals.

But, in fact, this means that industrial problems are defined as the necessity to solve problems that have to succeed in applying for many constrains, depending on different domains (mechanics, electronics, thermics, etc.). This tendency is quite recent and is focused on the opportunity to formalise some multi-domain constraints. This tendency needs some methods and tools to enable the resolution of complex problems and the first step is a modelling step. This is not trivial to model a multi-domain complex problem and recently some approaches and tools appear to give some interesting reply to this demand.

The difficulty is focused on the capacity of teaching this kind of training. How to imagine a course based on problem definition, and then problem-solving, in a reasonable time consuming ration? But, such kind of method and tool is one of the fundamental issues of the collaborative engineering teaching.

\subsection{The benefit of collaborative engineering software tools: the use of EDEN}

A collaborative engineering tool $\left(\mathrm{EDEN}^{\mathrm{TM}}\right)$, based on project's documents repository, has been set up. Documents are used to track the evolution of the project. The key concepts within the different formats of documents can be interrelated using master data. Good practice information is shared and experience and knowledge is captured within the EDEN environment. It ensures clarity, uniformity and coherency between different experts working on the same project. EDEN is an enterprise-wide innovation management tool used to support the collaborative modelling and the enterprise knowledge sharing (Candlot et al., 2004). 
This tool aims to re-use the existing structures of collaborative engineering work (RoadMaps). On the one side, the steps definitions guide the team into an already validated demarche. On the other side, each step is (can be) illustrated by previous best practices documents. Templates for the expected document to deliver can be found at each phase. Consequently, phase's objectives, performance indicators and ways to reach the goal are detailed in the RoadMap.

To get a detailed understanding and structuring of the project, the 'as-is' state can be modelled using appropriate modelling tools as well as an enterprise modelling architecture (Modified PERA (1996) in this case). This could be completed by the historic analysis (if existing).

As a result, improvements in the functions, information systems or organisational architecture can subsequently be developed using comprehensive project design life cycle architecture, thereby creating a required 'to-be' state of the project.

Figure 3 presents an example of the PERA Master Plan architecture that serves as a roadmap for the improvement effort. An enterprise-wide innovation management software tool called EDEN supports this improvement effort.

Figure 3 PERA master plan roadmap

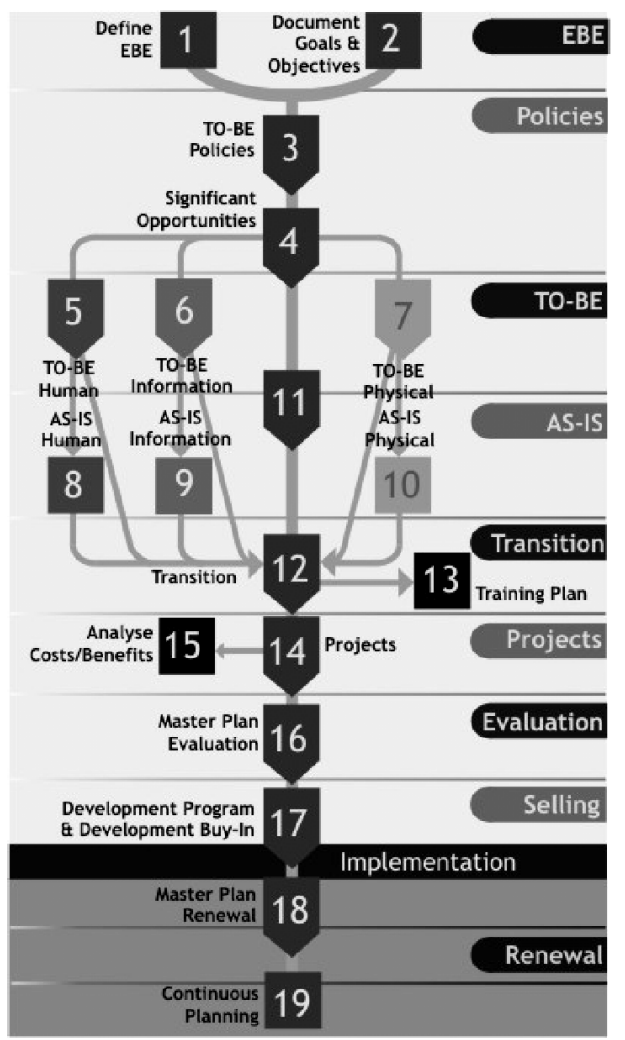

EDEN is a software environment, which provides high-level control over change-projects, supporting a multi-disciplinary team through a predefined roadmap structure (EDEN, 2006). It contains different enterprise reference architectures such as an 
enterprise life cycle, product life cycle, technology life cycle, and master plan for change projects that serve as a common reference framework and creating a unified understanding of the enterprise infrastructure. Figure 4 presents an example of the EDEN user interface.

Figure 4 EDEN user interface

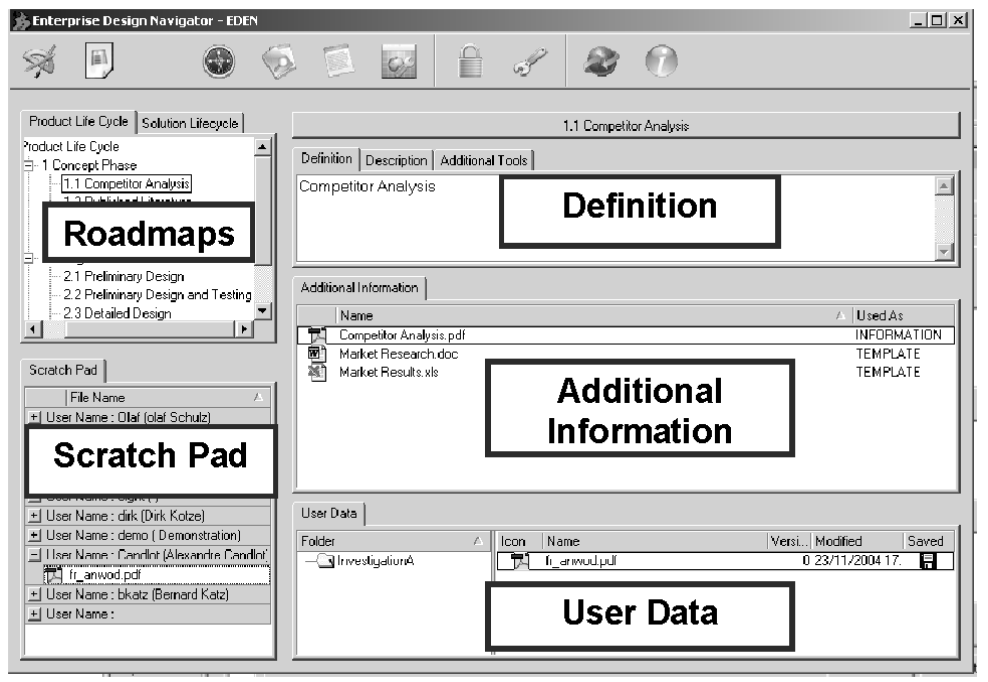

Knowledge repository using a document management tool will speed up both project life cycle and innovation loops.

\subsection{Interest of using such a collaborative engineering platform for project achievement during engineering education}

The use of collaborative engineering environments makes the students aware of the needs of:

- document procedure definition

- who is the author, who has right to modify/delete?

- modifications tracking, document versioning

- templates definition and use

- information sharing difficulties

- availability of knowledge inside the documents

- efficiency of data research within the mass

- data structure definition 
- collaborative approaches

- objectives and strategy definitions for everybody

- mutual understanding and definition between different fields of interests (expertise)

- global and dedicated views of the same problem

- knowledge and expertise sharing for innovative creation

- local validation within a global optimisation process.

On the other side, all the information system requirements can be presented for the students:

- access to the databases when the applications are located on a multi-site platform

- security of the data

- ownership of the data

- synchronisation between different sources.

\section{Necessity of a multi-domain approach and integration}

The actual trend, notably in industry, is to propose products more and more complex whose design spans over several engineering problems and disciplines. At the same time, enterprises have not only grown in complexity but also reduced their core competencies to be finally specialised in one (or several) discipline(s); thus, collaboration with subcontractors is now really common, even in the design phase. In addition, besides the traditional economic point of view, not only more recent industrial requirements, such as robustness and performance of the design, but also marketing criteria have appeared and quickly become important characteristics of the design and of its optimisation process. Nowadays, engineering design problems involve simultaneous optimisation of several objectives and satisfaction of constraints that have been determined by the design team. This is the multi-objective characteristic of the design problem, which is due to the nature of the designed product (or system). Besides, the global optimisation of the product - or the system - requires different competencies of several experts who may be located in different places and may work at different times. This is the multidisciplinary nature of the problem and depends on the enterprise's structure and decisions have to be taken quickly to be competitive.

\subsection{Decomposition of complex systems}

Within the work performed at IRCCyN and more especially in the Mechanical Design group (Giassi et al., 2004; Dépincé et al., 2005; Rabeau et al., 2006) we define complex systems as assemblies of interacting members that are difficult to understand as a whole (Allison et al., 2005) and the usual way to solve the optimisation problems that are linked to such systems is to decompose the problem into several sub-problems. 


\subsubsection{Several ways of decomposition}

In general, decomposition methodologies partitioning can be done in several ways such as object, aspect, sequential and model-based. Object decomposition divides a system by physical components. Aspect or discipline decomposition divides the system according to different disciplines - or specialities - and is relevant when multiple performance aspects are evaluated for a physical component. Object and aspect partitioning are 'natural' partitions and typically large companies employ both types of partitions simultaneously (mixed partition) in a matrix organisation (Rideout, 2001). Sequential decomposition is applicable when partitioned sub-problems are organised by workflow or process logic. Sequential decomposition presumes that the design information is unidirectional. Model decomposition is a more rigorous problem partitioning method based on functional dependencies between decision variables and functions included in the problem (Choudhary et al., 2005).

\subsubsection{Hierarchical decomposition}

According to the previous definition, complex systems are composed of several sub-systems, the so-called members. Frequently, one or several of these members are composed of sub-systems, and so on ... Such systems are called multilevel. Figures 5 and 6, respectively, show the structural decomposition of a theoretical system and of a real plane. The initial system (the plane) belongs to level zero. Level one is composed of three sub-systems in which several disciplines intervene. Then, each of these sub-systems could be partitioned into sub-systems, objects or disciplines. Such decomposition can be pursued until the basic members of the system appear. Actually, basic members are either components or disciplines that can more easily be optimised than the complex system in enterprises. Such decomposition is called hierarchical (Etman et al., 2005).

Figure 5 Structural decomposition of a multilevel system (see online version for colours)

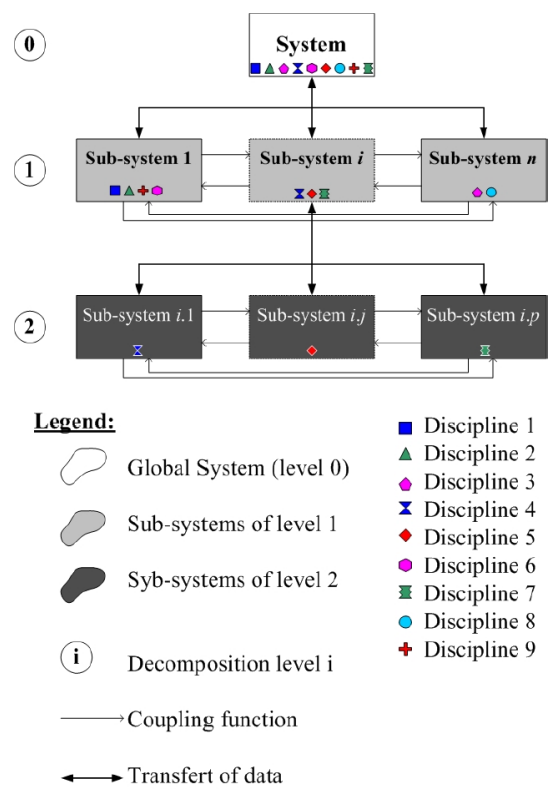


Figure 6 Example of a possible decomposition for a plane (see online version for colours)

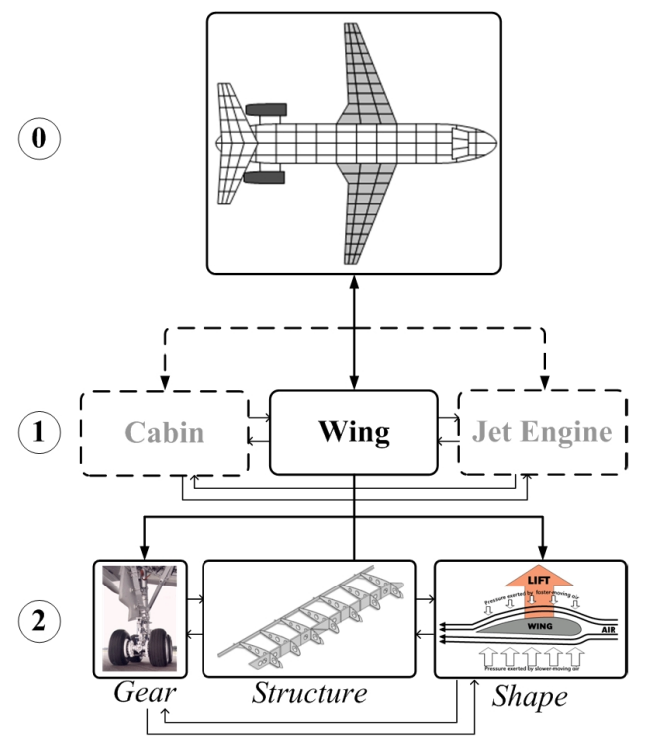

\subsubsection{Interaction between sub-systems}

Interactions between sub-systems exist at any level of the decomposition. However, they do not appear at the system level (level zero). Such interactions are usually called 'coupling functions'. They are output data for one of the sub-systems that are needed as input data by one or several other sub-systems. They ensure the system's coherence and synergy between members (Cramer et al., 1994; Balling and Sobieszczanski-Sobieski, 1996; Allison, 2004).

\subsubsection{Non-hierarchical systems}

Frequently, complex systems are non-hierarchical, which means that there is no reason to process the optimisation of one sub-system before another. In the optimisation process of such systems, the presence of these coupling functions and their consideration constitutes a real challenge for researchers.

\subsubsection{Problem resolution}

Two families of techniques exist to solve Multi Objective Optimization (MOO) problems: the first one consists in optimising (usually minimising) a unique function, which is constructed as an aggregation function. The problem is that such techniques lead to a unique solution (when it exists) that is strongly linked with the aggregation function. Consequently, the 'a priori' weight definition and aggregation function construction are major steps of the optimisation process. The main problem is to choose efficiently and justify the weights' choices of a function that has anyway no physical meaning. The second set of techniques provides a set of alternative optimal solutions. Over the years, the work of a considerable amount of operational researchers has produced an important number of techniques to deal with MOO problems. However, it was not until 
relatively recently that researchers realised the potential of evolutionary algorithms - or other population-based heuristics - in this area. The main motivation for using EAs in solving MOO problems rests on the fact that EAs deal simultaneously with a set of possible solutions (the so-called population), which allows us to find several members of the Pareto optimal set in a single run of the algorithm, instead of having to perform a series of separate runs as in the case of the traditional mathematical programming techniques (Multi-Disciplinarity Optimization (MDO)-Technical-Committee, 1991; Gu et al., 2002).

\subsection{Multidisciplinary approaches for the resolution of multi-objective problems}

\subsubsection{MDO in the extended enterprise}

The AIAA MDO Technical Committee has defined (MDO), in 1991, as a methodology for the design of complex engineering systems and sub-systems that coherently exploits the synergism of mutually interacting phenomena. System optimisation in the extended enterprise is usually divided into two main steps: analysis and optimisation.

Multidisciplinary analysis: Design requires an analysis of each component of the system to compute the system's characteristic functions (objective, constraint and coupling functions). Moreover, the analysis includes specific disciplinary knowledge and competencies such as experience, intuition, mathematical models, empirical results and computer simulations ... and such requirements imply the work of several specialised teams and/or subcontractors and/or companies that may be located in different places and work synchronously or asynchronously. They participate in the design in the context of the 'extended - distributed - enterprise' (Figure 7). All participants (teams, subcontractors, companies ...) collaborate on the design of a product according to their respective competencies. Each participant can be subdivided into several generic actors classified as departments, offices or designers. They use specific tools and the design of a product requires the action and input of the different actors and tools - software.

Figure 7 Context of the extended enterprise

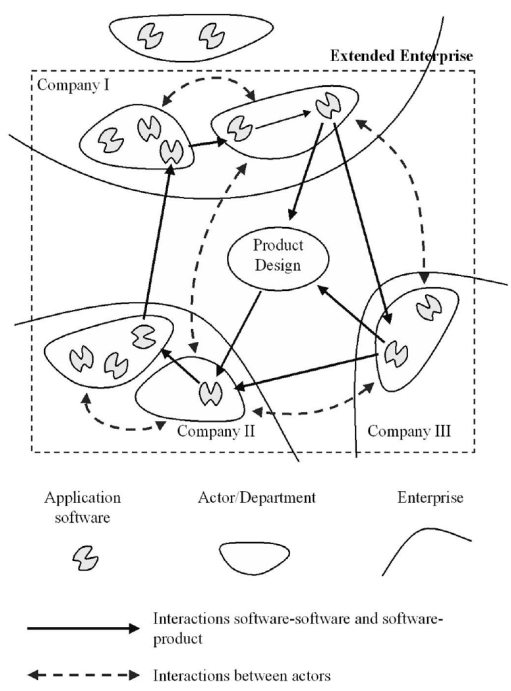


Multidisciplinary optimisation in the extended enterprise: to process the system design and optimisation, a lot of methodologies have been developed. They can be classified into two families: those which optimise the whole system and those which decompose the system into sub-systems to optimise sub-systems - disciplines - separately. The first set of methodologies is not adapted to the modern extended enterprise while the second one has been created especially to reach this goal and can conform to the enterprise tools, methodologies and software. In such a case, the multi-objective problem definition is adapted to be solved in the extended enterprise as depicted in Figure 8.

Figure 8 Multidisciplinary definition and structure of a multi-objective problem

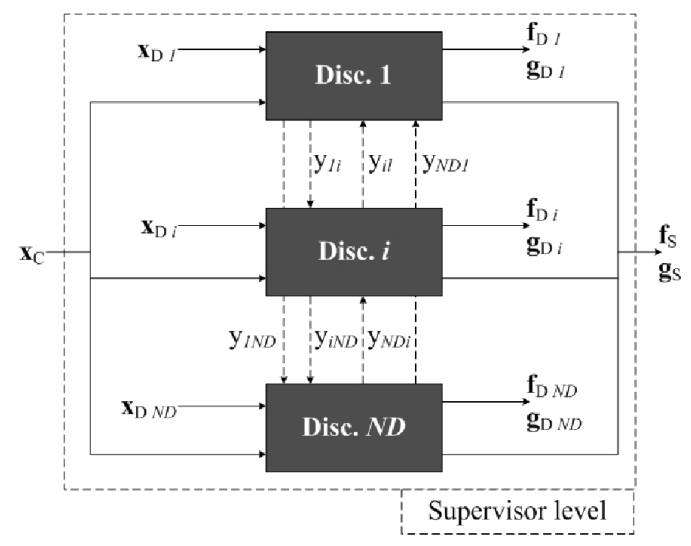

\subsubsection{Formulation of $M D O$ problem}

The corresponding formulation of the optimisation problem is modified as follows (Rabeau et al., 2006):

Find the set of design variables $\mathbf{x} \in$ DVS

that minimise objective functions $\mathbf{f} \in \mathrm{OFS}$ :

$$
\mathbf{f}(\mathbf{x})=\left(\mathbf{f}_{D_{1}}\left(\mathbf{x}_{D_{1}}, \mathbf{x}_{C}, \mathbf{y}_{j, 1}\right), \ldots, \mathbf{f}_{D_{\mathrm{NOD}}}\left(\mathbf{x}_{D_{\mathrm{NOD}}}, \mathbf{x}_{C}, \mathbf{y}_{j, \mathrm{NOD}}\right), \mathbf{f}_{S_{1}}(\mathbf{x}), \ldots, \mathbf{f}_{S_{\mathrm{NFS}}}(\mathbf{x})\right)^{T}
$$

and simultaneously satisfy equality and inequality constraints:

$$
\mathbf{g}(\mathbf{x})=\left(\mathbf{g}_{D_{1}}\left(\mathbf{x}_{D_{1}}, \mathbf{x}_{C}, \mathbf{y}_{j, 1}\right), \ldots, \mathbf{g}_{D_{\mathrm{NOD}}}\left(\mathbf{x}_{D_{\mathrm{NOD}}}, \mathbf{x}_{C}, \mathbf{y}_{j, \mathrm{NOD}}\right), \mathbf{g}_{S_{1}}(\mathbf{x}), \ldots, \mathbf{g}_{S_{\mathrm{NFS}}}(\mathbf{x})\right)^{T}
$$

where $\mathbf{x}_{\mathrm{D} i}$ is the set of design variables associated with discipline $i, \mathbf{x}_{C}$ is the set of common design variables, $\mathbf{f}_{\mathrm{D} i}$ is the set of objective functions associated with discipline $i, \mathbf{f}_{\mathrm{S} i}$ is the set of objective functions associated with the whole system, $\mathbf{g}_{\mathrm{D} i}$ is the set of constraint functions associated with discipline $i, \mathbf{g}_{\mathrm{S} i}$ is the set of constraint functions associated with the whole system (note that: $\mathbf{g}_{\mathrm{D} i}$ and $\mathbf{g}_{S i}$ can be equality or inequality constraints), $\mathbf{y}_{j i}$ is the set of coupling functions needed by discipline $i$ to compute objective and coupling functions, $j$ refers to disciplines that provide a coupling function to discipline $i, N O D$ is the number of disciplines, NFS is the number of system functions, $N G S$ is the number of system constraints.

At the disciplinary level, the problem induced by the previous formulation is the following, for each discipline $i$ : 
Find the set of local design variables

that minimise objective functions:

$$
\mathbf{f}_{D_{i}} \in \mathrm{OFS}: \mathbf{f}_{D_{i}}\left(\mathbf{x}_{D_{i}}, \mathbf{x}_{C}, \mathbf{y}_{j, i}\right)=\left(f_{D_{i_{1}}}, \ldots, f_{D_{i_{\mathrm{NOFi}}}}\right)
$$

and simultaneously satisfy equality and inequality constraints:

$$
\mathbf{g}_{D_{i}}\left(\mathbf{x}_{D_{i}}, \mathbf{x}_{C}, \mathbf{y}_{j, i}\right)=\left(g_{D_{i_{1}}}, \ldots, g_{D_{i_{\mathrm{NOF} i}}}\right) .
$$

In such cases, each team of the enterprise or each subcontractor is responsible for the design of one sub-set (sub-system) or a single facet of the product. These design tasks are assigned according to disciplinary distribution. It is important to note that the structural definition of the MDO problems is strongly linked to the methodology chosen to solve it (Cramer et al., 1994).

\subsubsection{Optimisation of complex systems in the extended enterprise}

The design of complex problems is not only a multi-objective process but also a multidisciplinary one. Such design problems are non-hierarchic (or coupled/networked problem), usually of large scale and characterised by large numbers of design variables and parameters, requirements - or constraints, and objective functions. Often, a high level of coupling between the system design participating disciplines exists, it is due to the information exchange that is required during the system analysis and evaluation: outputs of one discipline are inputs for other disciplines (Sobieszczanski-Sobieski and Haftka, 1997). This characteristic had led to the development of MDO processes.

\subsection{Tools and methods for MDO}

\subsubsection{MDO methodology families}

MDO methods can be summarised as methods that perform an analysis and optimisation for each sub-system, then at the system level an aggregation of these analyses is done that ensures the compatibility of the sub-systems. Different methods have been proposed and a review of them can be found in Sobieszczanski-Sobieski and Haftka (1997) and Kodiyalam and Sobieszczanski-Sobieski (2001).

The standard approach consists of performing an analysis module that is the integrated set of analysis models. For a given set of design variables, it returns the values of the constraints and the objective functions that are used by the optimiser module at the system level. This approach accommodates the interdisciplinary coupling within the integrated set of analysis models. One can note that this approach requires an iterative process during each evaluation of the objective functions, which ends when the convergence is reached. Other approaches belong to distributed architectures (All-At-Once (AAO), Concurrent Subspace Optimization (CSSO), Collaborative Optimization (CO) ...). Advantages of these architectures are both on organisational and computational levels (Braun et al., 1996) and (Alexandrov and Hussaini, 1995). Their organisational advantages with regard to standard approach include:

- more natural fit to current disciplinary expertise structure

- empowerment of the disciplinary experts in the design decision process 
- flexibility to alter a portion of the design without necessity to repose the complete problem.

Regarding the computational level, one can note:

- reduction in the integration requirements

- a parallel architecture that allows the use of heterogeneous platforms

- removal of iteration loops (coupling function)

- a reduced level of disciplinary sequencing.

Within the AAO optimisation approach, the analyses of each sub-system are executed all at once together. The optimiser has to ensure compatibility among coupling variables: an equality constraint and a design variable are added to the optimisation problem set. The requirement of producing a compatible multidisciplinary model becomes an additional task for optimisation. One drawback of this approach is that there is only one analysis at the sub-system level. However, frequently, it may be interesting to perform optimisation at this level. Discipline feasible constraint approaches such as CSSO or CO allow for greater authority among distributed sub-systems (Braun et al., 1996). The idea is that an optimisation is performed within each sub-system, the results that satisfy the disciplinary equations may not be interdisciplinary compatible. The main difference between CSSO and CO is that in the first one the system level is only a coordinator (no system level variables and objective functions). Within CO, each sub-system uses an independent optimiser. The objective of the system level is

- to find a compromise (coordination of the process between the disciplinary sub-systems)

- to satisfy the overall objective.

Furthermore, the system level optimiser is a method for arbitrating among coupled design variables. We have to underline the high computational cost of this approach. The fundamental idea behind $\mathrm{CO}$ is that the disciplinary experts should participate while not having to address local changes imposed by other experts.

\subsubsection{Commercial software}

Since five years, one can find some commercial software tools that allow to the process Integration and MDO. In some market, such as mechanical design automation market, it is one of the fastest growing segments.

The great majority of these tools allows one to

- Evaluate and compare several alternatives of the design (using techniques like sensibility and robustness analysis) by integrating different software (CAD, CAE, Matlab, ...)

- Provide design exploration and optimisation technology to ensure that an optimal design is discovered that meets or exceeds all customer requirements

- Determinate response surface model to spare calculus time, ... 
One can mention: ModeFrontier (Esteco), ModelCenter (Phoenix Integration), Isight (Engeneous Software), Optimus (Noesis Solutions).

\section{Discussion and conclusions}

In this paper, we have proposed an approach for the structuration of education curricula contents and methods based on project-based pedagogy, on the use of collaborative engineering platforms and on multi-domain optimisation methods and tools. These elements enable the student to be able to realise projects in a context similar to an extended enterprise. It is always difficult to structure and realise projects with students but the main problem they encounter is to manage time. As a matter of fact, they achieve a very complete work that can be transferred to another group for the following steps, the industrialisation of the product. If some other requirements appear, they have a complete structure that enables us to rapidly analyse and justify their strategy and their results, mainly due to the collaborative engineering platform and the multi-domain optimising system they use. In such context, the students are very motivated and they consider that this is for us a very fundamental practice that will be of benefit for the future.

Of course, some difficulties are still present. The main ones are the following:

- The goal definitions. Students focus on some a priori aspects of the projects and define sharply some targets and ways to reach them. But, some less obvious aspects, or not highlighted in the early presentation of the subject, are not as well defined. It results in difficulties to align all the expectations under a coherent and global hat. Some topics are not squared and the results hardly fit with the rest of the projects.

- The informatics system definition and building. Fears from the IS responsible combine with the high potentiality of non-conform use of the students makes the collaborative engineering platform design hard to set up. But collaboration means open possibilities to exchange, share or archive data. Without this easy and friendly use, the support of collaboration will be useless.

The next step will be to extend our experiences to different projects in several domains in collaboration with external teams of students, from other universities in the world who have the same tools and platforms. It may also favour a more systematic transfer of these kinds of tools, methods and approaches to the companies.

\section{References}

Alexandrov, N. and Hussaini, Y. (1995) 'Multidisciplinary design optimization: state of the art', in Alexandrov, N.M. and Hussaini, M.Y. (Eds.): Proceedings of the ICASE/NASA Langley Workshop on Multidisciplinary Design Optimization, 13-16 March, SIAM Publications, Hampton, Virginia.

Allison, J. (2004) Complex System Optimization: A Review of Analytical Target Cascading, Collaborative Optimization and Other Formulations, Master's Thesis, University of Michigan.

Allison, J., Kokkolaras, M. and Papalambros, P. (2005) 'On the impact of coupling strength on complex system optimization for single-level formulations', Proceedings of DETC'05 Long Beach, ASME Design Engineering Technical Conferences, California, USA. 
Balling, R. and Sobieszczanski-Sobieski, J. (1996) 'Optimization of coupled systems: a critical overview of approaches', AIAA, Vol. 34, No. 1, pp.6-17.

Braun, R., Cage, P., Kroo, I. and Sobieski, I. (1996) 'Implementation and performance issues in collaborative optimization', AIAA/NASA/ISSMO Symposium on Multidisciplinary Analysis and Optimization, Vol. 1, pp.295-305.

Candlot, A., Du Preez, N.D. and Bernard, A. (2004) 'Synergy and knowledge in an innovative project between academia and industry', International Conference on Competitive Manufacturing, ISBN 0-7972-1018-0, Stellenbosch.

Choudhary, R., Malkawi, A. and Papalambros, P. (2005) 'Analytic target cascading in simulation-based building design', Automation in Construction, Vol. 14, pp.551-568.

Cramer, E., Dennis, J.J., Frank, P., Lewis, R. and Shubin, G. (1994) 'Problem formulation for multidisciplinary optimization', SIAM Journal of Optimization, No. 4, pp.754-776.

Dépincé Ph., Rabeau S. and Bennis F. (2005) 'Collaborative optimization strategy for multi-objective design', ASME Int. Conf. on Design Engineering Technical Conferences and Computer and Information in Engineering Conference, 24-28 September, Long Beach, California.

EDEN (2006) EDEN Software, Indutech (Pty) Ltd, http://www.indutech.co.za/products/ software.htm

Etman, L., Kokkolaras, M., Hofkamp, A., Papalambros, P. and Rooda, J. (2005) 'Coordination specification in distributed optimal design of multilevel systems using the x language', Structural and Multidisciplinary Optimization Journal, Vol. 29, pp.198-212.

Giassi A., Bennis F. and Maisonneuve J.J. (2004) 'Multi-disciplinary design optimization and robust design approaches applied to concurrent design', Structural and Multidisciplinary Optimization Journal, Vol. 27, pp.1-16.

Gu, X., Renaud, J., Ashe, L., Batill, S., Budhiraja, A. and Krajewski, L. (2002) 'Decision-based collaborative optimization', Journal of Mechanical Design, Vol. 124, pp.1-13.

Kodiyalam, S. and Sobieszczanski-Sobieski, J. (2001) 'Multidisciplinary design optimization some formal methods, framework requirements, and application to vehicle design', International Journal of Vehicle Design (special issue), pp.3-32.

MDO-Technical-Committee (1991) Current State of the Art in Multidisciplinary Design Optimization, Technical Report, AIAA.

Pera (1996) Pera Master Planning Handbook, Purdue University, http://www.pera.net/ Arc_pera.html

Rabeau, S., Dépincé Ph. and Bennis, F. (2006) 'COSMOS: Collaborative optimization strategy for multi-objective systems', TMCE 2006, 18-22 April, Ljubljana, Slovenia.

Rideout, D.G., Stein, J.L., Ferris, J.B. and Hogland D.K. (2001) 'Target cascading: a design process for achieving vehicle ride and handling targets', Proceedings of the ASME IMECE Conference, Dynamic Systems and Control Division, Symposium on Advanced Automotive Technologies, DSC-9-4, New York, pp.1-11, http://www.mgfcar.de/hydragas/ geoff_DSC-9-4.pdf

Sobieszczanski-Sobieski, J. and Haftka, R. (1997) 'Multidisciplinary aerospace design optimization: survey of recent developments', Structural Optimization, Vol. 14, pp.1-23. 